\title{
Incidence of pathogens infections in a Romanian Intensive Care Unit and sensitivity to antibiotics. A prospective single center study
}

\author{
Bedreag Ovidiu Horea1,2, Rogobete Alexandru Florin ${ }^{1} 2^{\star}$, Luca Loredana1 ${ }^{1}$, Neamtu Claudiu ${ }^{1}$, Dragulescu \\ Dorian Mihai1, Nartita Radu1,3, Papurica Marius ${ }^{1,2}$ \\ ${ }^{1}$ Clinic of Anesthesia and Intensive Care, Emergency County Hospital "Pius Brinzeu", Timisoara, Romania \\ 2 Faculty of Medicine, "Victor Babes" University of Medicine and Pharmacy, Timisoara, Romania \\ 3 Faculty of Chemistry, Biology, Geography, West University of Timisoara, Timisoara, Romania
}

Introduction: Nosocomial infections represent one of the biggest challenges faced by clinicians in the intensive care unit (ICU) and is associated with high morbidity and mortality. Infections in ICU are most often very serious and represent often the cause of hospitalization in intensive care clinics.

Aim of the study: This paper presents the incidence of nosocomial infections, and the sensitivity to antibiotics encountered in our ICU.

Material and Methods: This prospective study was conducted for two years at the Clinic of Anesthesia and Intensive Care, Emergency County Hospital "Pius Brinzeu" Timisoara, Romania. All patients admitted to the ICU were analyzed in terms of signs and symptoms of bacterial infections.

Results: A total of 1081 microbiological reports were recorded. Among these, 635 (58.70\%) represented infections in the respiratory tract, $201(18.60 \%)$ in the bloodstream, $100(9.30 \%)$ in genitourinary tract, and $10(0.90 \%)$ in the central nervous system. The top five most frequently identified pathogen in microbiological reports are Klebsiella sp (17.60 \%), Acinetobacter sp (14.20 \%), Proteus mirabilis (13.80 \%), Pseudomonas aeruginosa (12.90 \%), Staphylococcus aureus - MSSA (12.80\%).

Conclusions: In order to choose empirical treatment, international guidelines should be consulted according to each pathology and adapted to the sensitivity encountered in the microbiology reports of the Critical Care Unit.

Keywords: pathogens, intensive care unit, antibiotics

Received: 30 October 2015 / Accepted: 05 January 2016

\section{Introduction}

Hospital acquired infections represent one of the biggest challenges faced by clinicians in the intensive care unit (ICU) in daily medical practice [1-3]. Diagnosis and treatment of infections with different locations and degrees of severity is one of the main tasks for medical specialists in intensive care. Also, sepsis remains a major cause of late mortality in trauma patients [4]. The onset of sepsis can occur with all its specific symptoms or, on the contrary, the clinical symptoms can be difficult to detect as they can be masked by underlying disease. Once there is a query of organ infection, one of the main goals is to identify the pathogen [5,6]. Typically, bacteriological diagnosis is made by cultures or Gram staining and microscopic identification. Before selecting an empiric antibiotic treatment, many clinical and patient-specific factors must be taken into account. Contemporary medicine based on extensive monitoring led to an increase of survival of patients, but also to an increase in number of days of hospitalization in the ICU. The use of invasive devices for monitoring and treatment, with the ones described above, creates circumstances for increased incidence of nosocomial infections [7]. The main goal of treatment is early and correct diag-

* Correspondence to: Alexandru Florin Rogobete

E-mail: alexandru.rogobete@umft.ro nosis of infection, as well as efficient antibiotic treatment that should be started as soon as possible [8]. Lack of an effective antibiotic treatment in the first hours of admission decreases the chances of survival and studies showed that initiation of appropriate treatment after 48 hours of diagnosis is virtually useless and mortality is almost $100 \%$ for some severe life-threatening infections [7-11]. Thus arose the concept of de-escalation antibiotic therapy - early initiation of ultra-broad-spectrum antibiotic therapy that cover potential pathogens incriminated in the occurrence of sepsis. When sensitivity is available, antibiotics will be readjusted accoriding to the results (hence the origin of the term de-escalation - it starts from an ultra-coverings antibiotic therapy subsequently restricting the antiobiotics depending on the sensitivity).

Because antibiotic sensitivity results are validated after at least 48-72 hours, it becomes obvious that understanding the types of germs most frequently incriminated in some infectious diseases can be extremely beneficial to allow a more accurate therapy in the earliest hours of admission $[2,9,12-14]$.

In this paper, we want to present the microbiological reports in our ICU, comprising the incidence of infections reported to total population, the incidence of various types of infections (respiratory system / urogenital tract / central nervous system / abdominal infections / bloodstream in- 
fections etc.), types of pathogens related to the source of infection, the overall incidence of pathogens and antibiotic sensitivity analysis of pathogens.

\section{Methods}

\section{Study design}

This prospective study was conducted from 1st of January 2013 - 1st of January 2015 in Anaesthesia and Intensive Care Clinic of Emergency County Hospital of „Pius Brinzeu” Timisoara, Romania. Database of the study was obtained by entering data into the database of the hospital. All information has been used by respecting the informed consent of the hospital ethics committee.

\section{Patient population}

All patients admitted in ICU in Emergency County Hospital "Pius Brinzeu" Timisoara, between 1 January 2013 and 1 January 2015 were analyzed. Regarding microbiological reports, a series of criteria were analyzed, such as the source of infection, the incidence of pathogens identified in each source / system, antibiotic sensitivity of each pathogen. Microbiological analyzes were performed in the Microbiology Laboratory of Clinical Emergency Hospital "Pius Brinzeu" Timisoara.

\section{Statistical analysis}

The statistical analysis performed in the study consisted in the calculation of frequencies and percentages, as well as the calculation of the arithmetic means and the standard deviations. Unpaired $t$ test was used for variables expressed as the mean and Chi square test for those expressed in percentages. Statistical significance was defined as $\mathrm{p}<0.05$. Statistical analysis of data was performed with the computer software Prism 6 for Mac OS X v.6.0. (GraphPad Software, Inc., San Diego, CA) and Microsoft Office Excel for Mac 2011 v.14.4.8. (Microsoft Corporation, Bucharest, Romania).

\section{Results}

1081 microbiology reports were analyzed. Table I presents the number of microbiology reports and the incidence accourding to the original sourse of infection. The most common organ dysfunction in ICU is respiratory failure (usually with mechanical ventilation), having as main causes: coma, multiple traumas, sepsis. Typically, over $80 \%$ of patients admitted to ICU are intubated and mechanically ventilated. This inevitably leads to increased incidence of

Table I. Microbiology reports related to the source

\begin{tabular}{lcc}
\hline Source & No. of microbiology reports & Percent \\
\hline Respiratory tract & 635 & $58.70 \%$ \\
Bloodstream & 201 & $18.60 \%$ \\
Genito-urinary tract & 100 & $9.30 \%$ \\
Abdomen & 92 & $8.50 \%$ \\
CNS & 10 & $0.90 \%$ \\
Others & 43 & $4 \%$ \\
\hline
\end{tabular}

pulmonary infections which are, as indicated by the statistics obtained, the most frequent infectious problems encountered in the ICU of our hospital.

Table II presents the number of cases confirmed by positive cultures and their incidence depending on the source. There is an increased incidence of germs in the top four positions (Klebsiella sp., Acinetobacter sp., Proteus mirabilis, Pseudomonas aeruginosa), with a rate of about 16$17 \%$ each, followed immediately by Staphyloccocus aureus MSSA with a percentage of $13.6 \%$. The next most frequent pathogen agents are Staphyloccocus aureus MRSA and E. Coli. Streptococcus pneumoniae, Streptococcus group A, B, C, G have a lower incidence and usually are responsible for development of community-acquired pneumonia. Haemophilus influenza was not identified, which is a bacteria commonly incriminated in the occurrence of early onset nosocomial pneumonia or early onset of ventilator associated pneumonia.

The conclusion of this data is that differentiation of nosocomial pneumonia by the time of onset and the supposition that early onset pneumonias are caused by community agents with good sensitivity to common antibiotics, cannot be applied in our clinic as the majority of nosocomial pneumonias are caused by agents with potential multiresistance to antibiotics. The overall incidence in ICU, regardless of the source of infection is represented by: 17.60 $\%$ Klebsiella sp (E. Coli/Klebs sp ESBL, E. Coli/Klebs sp KPC), $14.20 \%$ Acinetobacter sp., $13.80 \%$ Proteus mirabillis, $12.90 \%$ Pseudomonas Aeruginosa, 12.80 \% Staphyloccocus aureus (MSSA), $8.30 \%$ E. Coli (E. Coli/Klebs sp ESBL, E. Coli/Klebs sp KPC), 6.90 \% Staphyloccocus aureus (MRSA), $5.10 \%$ Candida albicans, $2.20 \%$ Providencia sp, $1.50 \%$ Staphyloccocus aureus (CA-MRSA), $0.70 \%$ Enterococcus faecalis, $0.60 \%$ Streptococcus group A, B, C, G, $0.50 \%$ Serratia sp, $0.40 \%$ Enterobacter sp, $0.20 \%$ Streptococcus pneumonie, $0.20 \%$ Proteus vulgaris, $0.10 \%$ Candida parapsilosis, $0.10 \%$ Citrobacter sp.

The most frequent infectious pathology is located in the respiratory tract, followed at a great distance by bloodstream infections and those caused by the presence of central venous catheters.

\section{Discussions}

International guidelines noted that Klebsiella species have intrinsic resistance to ampicillin and other aminopenicillins, and develop resistance to cephalosporins and aztreonam by producing extenden-spectrum beta-lactam [15]. Also, they can develop resistance to aminoglycosides and other antibiotics, but remain sensitive to carbapenems [16,17]. Resistance to cephalosporins and aminoglycosides is well known in south and east Europe. Resistance to carbapenems is induced by the production of metallo-betalactamases capable of hydrolyzing the antibiotic molecule $[1,15]$.

Based on the above, we note that the strains of Klebsiella sp. isolated in our ICU have unusually high resistance 
Table II. The incidence of pathogens identified by their source

\begin{tabular}{|c|c|c|c|c|c|}
\hline \multirow{2}{*}{ Pathogen agent } & \multicolumn{5}{|c|}{ Number of cases identified by positive culture (incidence \%) } \\
\hline & Respiratory tract & Genito-urinary tract & Intraabdominal & CNS & Bloodstream \\
\hline $\begin{array}{l}\text { Klebsiella sp. (E. coli/Klebs sp ESBL, E. coli/ } \\
\text { Klebs sp KPC) }\end{array}$ & $124(19.80)$ & $34(35.40)$ & $10(11.40)$ & $2(20)$ & $12(6.30)$ \\
\hline Acinetobacter sp. & $105(16.80)$ & $7(7.30)$ & $8(9.10)$ & $3(30)$ & $18(9.50)$ \\
\hline Proteus mirabilis & $98(15.70)$ & $16(16.70)$ & $8(9.10)$ & $1(10)$ & $17(8.90)$ \\
\hline Pseudomonas aeruginosa & $91(14.50)$ & $11(11.50)$ & $12(13.60)$ & $1(10)$ & $15(7.90)$ \\
\hline Staphyloccocus aureus (MSSA) & $85(13.60)$ & $0(0)$ & $11(12.50)$ & $3(30)$ & $27(14.20)$ \\
\hline Staphyloccocus aureus (MRSA) & $44(7)$ & $0(0)$ & $3(3.40)$ & $0(0)$ & $26(13.70)$ \\
\hline $\begin{array}{l}\text { E. coli (E. coli/Klebs sp ESBL, E. coli/Klebs } \\
\text { sp KPC) }\end{array}$ & $34(5.40)$ & $13(13.50)$ & $24(27.30)$ & $0(0)$ & $0(0)$ \\
\hline Providencia sp. & $15(2.40)$ & $0(0)$ & $1(1.10)$ & $0(0)$ & $8(4.20)$ \\
\hline Candida albicans & $8(1.30)$ & $10(10.40)$ & $4(4.50)$ & $0(0)$ & $30(15.80)$ \\
\hline Streptoccocus A, B, C, G & $5(0.80)$ & $0(0)$ & $1(1.10)$ & $0(0)$ & $0(0)$ \\
\hline Staphyloccocus aureus (CA-MRSA) & $3(0.50)$ & $0(0)$ & $1(1.10)$ & $0(0)$ & $9(4.70)$ \\
\hline Streptoccocus pneumoniae & $2(0.30)$ & $0(0)$ & $0(0)$ & $0(0)$ & $0(0)$ \\
\hline Proteus vulgaris & $1(0.20)$ & $0(0)$ & $1(1.10)$ & $0(0)$ & $0(0)$ \\
\hline Enterobacter sp. & $1(0.20)$ & $1(1)$ & $0(0)$ & $0(0)$ & $0(0)$ \\
\hline Serratia sp. & $1(0.20)$ & $1(1)$ & $0(0)$ & $0(0)$ & $0(0)$ \\
\hline Staphyloccocus epidermidis & $1(0.20)$ & $0(0)$ & $1(1.10)$ & $0(0)$ & $6(3.50)$ \\
\hline Citrobacter sp. & $0(0)$ & $1(1)$ & $0(0)$ & $0(0)$ & $0(0)$ \\
\hline
\end{tabular}

to third generation cephalosporins (over $60 \%$ of strains), high resistance to aminoglycosides (over $50 \%$ of strains) and fluoroquinolones (levoflaxacin has the best sensitivity, but active against only $60 \%$ of strains). Resistance to carbapenems occurs in over $30 \%$ of cases, and the sensitivity is as follows: ertapenem (61\%), imipenem (67\%) and meropenem (73\%). In vitro sensitivity to Colistin is $100 \%$, but this might not be reflected in vivo. Also, the results are influenced by the fact that Colistin sensitivity was not tested on a regular basis. Among aminoglycoside, amikacin and tobramycin are worth mentioning as they show acceptable sensitivity (69\% for tobramycin), but unfortunately are not available in the pharmaceutical market in Romania.

Acinetobacter sp. is a Gram negative coccobacillus, strictly aerobic, non-fermentative, immobile, oxidasenegative. These agents are involved in nosocomial infections in immunocompromised patients $[18,19]$. The most clinically relevant family is represented by Acinetobacter calcoaceticus-baumanii complex [20]. An important characteristic is that they are highly resistant to antibiotics. There is a discrepancy regarding the sensitivity to carbapenems of certain strains, which can be variable. The clinical impact of this discrepancy was described in a case report by Lesho et al [21], when, based on premises that Acinetobacted Sp is sensitive to Imipenem, it was decided to continue the antibiotic treatment with meropenem, but eventually cultures showed that the strain was resistant to meropenem. Sensitivity for meropenem and imipenem varies between $30-70 \%$ with large differences depending on the location where the research was conducted [22]. Unfortunately, in our case, statistical analysis reveals extremely low sensitivity of Acinetobacter strains both to meropenem (16\%) and to imipenem (13\%). International guidelines recommended that in order to obtain a minimum effective inhibitory concentration for a longer period of time, doses of carbapenems should be given in continuous infusion of about 3 hours (due to half time of about $4 \mathrm{~h}$ for meropenem and imipenem, but not for doripenem which has a much longer half-life) [23]. Reintroduction of colistin in clinical practice brings notable benefits in the treatment of Acinetobacter sp. infections. Nephrotoxicity and neurotoxicity are its main adverse effects, but the risk / benefit balance tilts in favour of the administration of this polymixins [24]. The in vitro sensitivity to colistin of Acinetobacter sp. strains isolated in our case is $97 \%$.

Sulbactam is also known to have a bactericidal effect on Acinetobacter sp. In our statistics, $50 \%$ were susceptible to sulbactam present in combination ampicillin / sulbactam. Among aminoglycoside, only amikacin showed noteworthy efficiency of about $46 \%$.

Proteus Mirabilis is one of the most common Gramnegative agents and can cause a wide range of nosocomial or community infections, including urinary tract, respiratory, wounds or bloodstream infections [25-27]. This micro-organism is intrinsically resistant to nitrofurantoin and tetracycline but susceptible to $\beta$-lactam, aminoglycosides, trimethoprim-sulfamethoxazole and fluoroquinolones [28]. However, there is an increasing resistance of these species to broad spectrum cephalosporins due to the production of extended-spectrum beta-lactamases. In recent years, positive extended spectrum beta-lactamases strains of P. mirabilis have been described globally, with higher prevalence in certain geographic areas of Europe [29]. Genes encoding extended-spectrum beta-lactamases are usually located on transferable plasmids and are generally mutated genes of TEM-1/2 $\beta$-lactamase. More than that, positive extended-spectrum beta lactamases strains-PM are co-resistant to aminoglycosides, trimethoprim-sulfamethoxazole and fluoroquinolones [30]. In these cases, Europe- 
an guidelines recommend administration of carbapenems as the only therapeutic alternative [31].

In our patients, the most common strains of Proteus Mirabilis isolated from the respiratory tract are producing extended-spectrum beta-lactamases. Only $20 \%$ of strains are susceptible to ceftriaxone and only $10 \%$ are susceptible to ceftazidime (3rd generation cephalosporins). Trimethoprim-sulfamethoxazole sensitivity is 0 . Fluoroquinolones maintain a variable sensitivity (ciprofloxacin about 60\% and $67 \%$ for levofloxacin). The most important option for therapy remain carbapenem with a very good sensitivity (90\%) both to imipenem and meropenem. Also aztreonam is worth mentioning, with $100 \%$ efficiency.

Pseudomonas aeruginosa is one of the most common Gram-negative aerobic bacteria responsible for severe nosocomial infections. It is a multi-drug resistant germ, which greatly complicates the choice of antibiotic therapy [32-35]. Pseudomonas aeruginosa infections usually occur in immunocompromised patients or in anatomical sites with damaged natural barriers (skin, mucous membranes, etc.) and extremely rare in healthy people and are considered opportunistic infections [36].

Pseudomonas aeruginosa is intrinsically resistant to many antibiotics most commonly mediated by efflux pumps. Piperacillin, anti pseudomonas cephalosporin (ceftazidime, cefepime), other $\beta$-lactams, carbapenems (imipenem and meropenem), aminoglycosides and fluoroquinolones resistance are increasing globally. There is also an increase in the incidence of strains producing $\beta$-lactamases metal-mediated plasmids, active against carbapenems, cephalosporins and penicillins anti-pseudomonas [12,37]. The results of microbiological analysis on Pseudomonas aeruginosa isolated from the respiratory tract in our patients reveals great sensitivity to antipseudomonas aminoglycosides (amikacin, tobramycin - over 90\%), but unfortunately a poor sensitivity to carbapenems (imipenem and $52 \%$ to $46 \%$ for meropenem), slightly better for ceftazidime (56\%), tazobactam / piperacillin (56\%) and ciprofloxacin $(58 \%)$. From these data it is evident that the treatment of infections caused by Pseudomonas aeruginosa must be based on a combination of antibiotics followed my de escalation. In addition, antibiotic therapy should be increased to 14 days.

Staphyloccocus aureus MSSA does not cause special problems for intensive care physician, mainly because escalating therapy often used in the first phase of treatment is active against strains of Staphyloccocus aureus MSSA [38-39]. It should be noted that only when culture results become available, de-escalation should be performed promptly.

Staphyloccocus aureus MRSA represent a global problem, being resistant to common antistaphylococcal therapy $[40,41]$.

Resistance is caused by the production of a penicillin binding protein, encoded by the mecA gene. Although
Staphyloccocus aureus MSSA germ was cited with intermediate sensitivity to vancomycin (MIC $8-16 \mu \mathrm{g} / \mathrm{ml}$ ) and others with increased resistance (MIC 32-1024 mg/ml), there is no data that they are involved in respiratory tract infections [40,42-44]. In our clinic, isolated strains have good sensitivity to vancomycin, linzolid, teicoplanin and tygeciclin. There were no reported vancomycin-resistant / intermediate strains.

Escherichia Coli is a species of Gram-negative bacilli, single or pairs, which can cause a broad range of infectious diseases from urinary tract infections to nosocomial pneumonia. Escherichia genus was named after its discoverer, Theodor Escherich. Usually respiratory tract infections caused by E coli occur afte micro-aspirations from upper airways [45-47]. Co-morbidities such as diabetes, chronic obstructive bronchopneumonia, alcoholism favour E. coli infection. Clinically, bronchopneumonia usually affects the lower lobes, and can be complicated by empyema. Another means of dissemination is hematogenic, urogenital or abdominal source, in these cases bacteraemia with $\mathrm{E}$. coli preceding the occurrence of pneumonia [46-49].

Strains of E. coli isolated from the respiratory tract of our patients have a good sensitivity to the usual antibiotics. There were no reported cases of carbapenem-resistant strains. Resistance to cephalosporins third generation is also low.

Providencia include gram-negative bacilli producing urease, responsible for a wide range of nosocomial or community infections. While urinary tract is the most affected, Providencia may be associated with gastroenteritis, bacteraemia etc. Providencia infections are usually a problem for the clinician due to increased resistance to antibiotics caused by production of extended-spectrum beta-lactamases [50-52].

Providencia species are always resistant to tetracyclines, penicillins and first generation cephalosporins, but are sensitive to last generation cephalosporins, aztreonam, imipenem, meropenem. Providencia sp. have a variable sensitivity to fluoroquinolones, aminoglycosides and trimethoprim-sulfamethoxazole [51,53].

Providencia stuartii is typically the most resistant of all species of Providencia. An Italian study from 2006 showed that Providencia stuartii positive for extendedspectrum beta-lactamaze accounted for $10 \%$ of all strains of bacteria producing extended-spectrum beta-lactamases, and showed resistance to amoxicillin-clavulanate (81.8\%) ampicillin-sulbactam (40.1\%), gentamicin (79.5\%) and ciprofloxacin (84.1\%). In other sudiu, $53 \%$ of P stuartii strains produced extended-spectrum beta-lactamase [53]. The strains isolated from the respiratory tract in our clinic (15 strains) confirms data from the international literature. Carbapenems, amikacin, piperacillin / tazobactam and aztreonam show, in this order, the best sensitivity. Among carbapenems, ertapenem has very good sensitivity, usually used to treat community pneumonia. 
In order to choose empirical treatment, guidelines should be consulted depending on the source of infection and sensitivities as highlighted in the current study, as follows: the empirical treatment of infections of the bloodstream must be compulsory directed against: Staphylococcus aureus MSSA (methicillin sensitive) / Candida albicans / Staphylococcus aureus MRSA (methicillin resistant) / Acinetobacter and is recommended to include the combination of: vancomycin, colistin and antifungal; empiric treatment in the low urinary tract infections should be compulsory directed against: Klebsiella sp. / Proteus Mirabilis / Candida albicans / Pseudomonas aeruginosa and is recommended to include the combination of meropenem / imipenem and antifungal; empirical treatment of nosocomial pneumonia should be compulsory directed against: Klebsiella sp. / Acinetobater sp. / Proteus Mirabilis / Pseudomonas aeruginosa and is recommended to include the combination of: meropenem / imipenem, colistin and amikacin; empiric treatment in abdominal infections should be compulsory directed against E. coli / Klebsiella sp. / Methicillin-sensitive Staphylococcus and is recommended to be performed with meropenem / imipenem and aminoglycosides.

There is a low incidence of infections caused by community agents with good sensitivity to common antibiotics. This fact is due in part by the fact that the majority of the patients admitted in the ICU have a background of antibiotic administration, either started on the wards, or at home, leaving only the remaining resistant microbial flora.

\section{Acknowledgements}

The authors wish to thank the Microbiology Laboratory of the Emergency County Hospital "Pius Brinzeu” Timisoara to support this study.

\section{Conflict of interest}

The authors declare that they have no conflict of interest.

\section{References}

1. Sękowska A, Gospodarek E, Kusza K. The prevalence of infections and colonisation with Klebsiella pneumoniae strains isolated in ICU patients. Anaesthesiol Intensive Ther. 2014;46:280-283.

2. Różańska A, Bulanda M. Demographic characteristics of patients and their assessment of selected hygienic practices of hospital personnel in the context of safety climate of hospitalization. Am J Infect Control. 2015;43:354-357.

3. Singh S, Goyal R, Ramesh GS, et al. Control of hospital acquired infections in the ICU: A service perspective. Med Journal Armed Forces India. 2015;71:28-32.

4. Sevransky JE, Martin GS, Shanholtz C, et al. Mortality in sepsis versus non-sepsis induced acute lung injury. Crit Care. 2009;13:R150.

5. Coelho FR, Martins JO. Diagnostic methods in sepsis: the need of speed. Rev Assoc Med Bras. 2012;58:498-504.

6. Singh S, Chaturvedi R, Garg SM, Datta R, Kumar A. Incidence of healthcare associated infection in the surgical ICU of a tertiary care hospital. Med Journal Armed Forces India. 2013;69:124-129.

7. Dereli N, Ozayar E, Degerli S, Sahin S, Koç F. Three-Year Evaluation of Nosocomial Infection Rates of the ICU. Rev Bras Anestesiol. 2013;63:7384

8. Harwan WA, Abbassi MM, El-Attar MM, Farid SF. Pharmacoeconomic study of antibiotics used in the treatment of lower respiratory tract infections in ICU patients: A case study in an Egyptian hospital. Bull Fac
Pharmacy. 2014;52:135-144

9. Scherbaum M, Kösters K, Mürbeth RE, et al. Incidence, pathogens and resistance patterns of nosocomial infections at a rural hospital in Gabon. BMC Infect Dis. 2014;14:124.

10. Guggenbichler J, Assadian O, Kramer M. Incidence and clinical implication of nosocomial infections associated with implantable biomaterials - catheters, ventilator-associated pneumonia, urinary tract infections. GMS Krankenhhyg Interdiszip. 2011;6:1-19.

11. McDonald Cl, Fung YL, Shekar K, et al. The impact of acute lung injury, ECMO and transfusion on oxidative stress and plasma selenium levels in an ovine model. J Trace Elem Med Biol. 2015;30:4-10.

12. Mérens A, Delacour $H$, Plésiat $P$, Cavallo J-D, Jeannot K. Pseudomonas aeruginosa et résistance aux antibiotiques. Rev Francoph des Lab. 2011;2011:49-62.

13. Mahafza T, Batarseh S, Bsoul N. Early vs. late tracheostomy for the ICU patients: Experience in a referral hospital. Saudi J Anaesth. 2012;6:152154.

14. Juayang AC, Jr DGM, Reyes GBDL, Acosido MAD, Gallega CT. Review on the Antimicrobial Resistance of Pathogens from Tracheal and Endotracheal Aspirates of Patients with Clinical Manifestations of Pneumonia in Bacolod City in 2013. International Journal of Bacteriology. $2015 ; 2015$.

15. Gupta A, Ampofo K, Rubenstein D, Saiman L. Extended spectrum beta lactamase-producing Klebsiella pneumoniae infections: a review of the literature. J Perinatol. 2003;23:439-443.

16. Shigemoto N, Kayama S, Kuwahara R, et al. A novel metallo- $\beta$ lactamase, IMP-34, in Klebsiella isolates with decreased resistance to imipenem. Diagn Microbiol Infect Dis. 2013;76:119-121.

17. Charrouf FO, Hamze M, Mallat H, Achkar M, Dabboussia F. Characterization of resistance genes in 68 ESBL-producing Klebsiella pneumonia in Lebanon. Médecine Mal Infect. 2014;44:535-538.

18. Murray CK, Hospenthal DR. Acinetobacter infection in the ICU. Crit Care Clin. 2008;24:237-248.

19. Vourli S, Frantzeskaki F, Meletiadis J, et al. Synergistic interactions between colistin and meropenem against extensively drug-resistant and pandrug-resistant Acinetobacter baumannii isolated from ICU patients. Int J Antimicrob Agents. 2015;45:670-671.

20. Dueñas Díez Al, Bratos Pérez MA, Eiros Bouza JM, et al. Susceptibility of the Acinetobacter calcoaceticus-A. baumannii complex to imipenem, meropenem, sulbactam and colistin. Int $J$ Antimicrob Agents. 2004;23:487-493.

21. Lesho E, Wortmann G, Moran K, Craft D. Fatal Acinetobacter baumannii infection with discordant carbapenem susceptibility. Clin Infect Dis. 2005;41:758-759.

22. Ikonomidis A, Pournaras S, Maniatis AN, Legakis NJ, Tsakris A. Discordance of meropenem versus imipenem activity against Acinetobacter baumannii. Int J Antimicrob Agents. 2015;28:376-377.

23. Jaruratanasirikul S, Sriwiriyajan S, Punyo J. Comparison of the pharmacodynamics of meropenem in patients with ventilator-associated pneumonia following administration by 3-hour infusion or bolus injection. Antimicrob Agents Chemother. 2005;49:1337-1339.

24. Wood GC, Hanes SD, Croce M a, Fabian TC, Boucher B a. Comparison of ampicillin-sulbactam and imipenem-cilastatin for the treatment of acinetobacter ventilator-associated pneumonia. Clin Infect Dis. 2002;34:1425-1430.

25. Yang $\mathrm{Q}$, Zhang $\mathrm{H}$, Cheng $\mathrm{J}$, et al. In vitro activity of flomoxef and comparators against Escherichia coli, Klebsiella pneumoniae and Proteus mirabilis producing extended-spectrum $\beta$-lactamases in China. Int J Antimicrob Agents. 2015;45:485-490.

26. Kanayama A, Kobayashi I, Shibuya K. Distribution and antimicrobial susceptibility profile of extended-spectrum $\beta$-lactamase-producing Proteus mirabilis strains recently isolated in Japan. Int $J$ Antimicrob Agents. 2015;45:113-118.

27. Dzutsev A, Trinchieri G. Proteus mirabilis: The Enemy Within. Immunity. 2015;42:602-604.

28. O'Hara CM, Brenner FW, Miller JM. Classification, identification, and clinical significance of Proteus, Providencia, and Morganella. Clin Microbiol Rev. 2000;13:534-546.

29. Nijssen S, Florijn A, Bonten MJM, et al. Beta-lactam susceptibilities and prevalence of ESBL-producing isolates among more than 5000 European Enterobacteriaceae isolates. Int J Antimicrob Agents. 2015;24:585-591.

30. De Champs C, Bonnet R, Sirot D, Chanal C, Sirot J. Clinical relevance of Proteus mirabilis in hospital patients: a two year survey. J Antimicrob Chemother. 2000;45:537-539.

31. Stürenburg E, Mack D. Extended-spectrum $\beta$-lactamases: implications for the clinical microbiology laboratory, therapy, and infection control. J Infect. 2015;47:273-295. 
32. Hofmann A, Sommer R, Hauck D, et al. Synthesis of mannoheptose derivatives and their evaluation as inhibitors of the lectin LecB from the opportunistic pathogen Pseudomonas aeruginosa. Carbohydr Res. 2015;412:34-42.

33. Sahner JH, Empting M, Kamal A, et al. Exploring the chemical space of ureidothiophene-2-carboxylic acids as inhibitors of the quorum sensing enzyme PqsD from Pseudomonas aeruginosa. Eur J Med Chem. 2015;96:14-21.

34. Potron A, Poirel L, Nordmann P. Emerging broad-spectrum resistance in Pseudomonas aeruginosa and Acinetobacter baumannii: Mechanisms and epidemiology. Int J Antimicrob Agents. 2015;45:568-585.

35. Michalopoulos a. S, Tsiodras S, Rellos K, Mentzelopoulos S, Falagas $\mathrm{ME}$. Colistin treatment in patients with ICU-acquired infections caused by multiresistant Gram-negative bacteria: The renaissance of an old antibiotic. Clin Microbiol Infect. 2005;11:115-121.

36. Frédéric $\mathrm{G}$, Julien $\mathrm{T}$, Benoit $\mathrm{R}$, et al. Selective digestive decontamination and empirical antimicrobial therapy of late - Onset ventilator - Associated pneumonia in trauma patients. J Acute Dis. 2012;1:1-6.

37. Cohen MJ, Block CS, Moses AE, Nir-Paz R. Exposure to ertapenem is possibly associated with Pseudomonas aeruginosa antibiotic resistance. Clin Microbiol Infect. 2014;20:0188-196.

38. Van der Wolf PJ, Rothkamp A, Junker K, de Neeling AJ. Staphylococcus aureus (MSSA) and MRSA (CC398) isolated from post-mortem samples from pigs. Vet Microbiol. 2012;158(1-2):136-141.

39. Paul M, Zemer-Wassercug N, Talker O, et al. Are all beta-lactams similarly effective in the treatment of methicillin-sensitive Staphylococcus aureus bacteraemia? Clin Microbiol Infect. 2011;17:1581-1586.

40. Patel H, Nguyen GC. S1103 Association of Methicillin-Resistant Staph Aureus (MRSA) with Higher Mortality Among Hospitalized IBD Patients. Gastroenterology. 2009;136:A - 189.

41. Köck R, Winner K, Schaumburg F, et al. Admission prevalence and acquisition of nasal carriage of meticillin-resistant Staphylococcus aureus (MRSA) in German rehabilitation centres. J Hosp Infect. 2014;87:115118.

42. González-Domínguez M, Seral C, Sáenz Y, et al. Epidemiological features, resistance genes, and clones among community-onset methicillinresistant Staphylococcus aureus (CO-MRSA) isolates detected in northern Spain. Int J Med Microbiol. 2012;302:320-326.
43. Goodwin KD, McNay M, Cao $Y$, et al. A multi-beach study of Staphylococcus aureus, MRSA, and enterococci in seawater and beach sand. Water Res. 2012;46:4195-4207.

44. Rao N, Bennett JE. Eradication of methicillin resistant Staph aureus (MRSA) with ciprofloxacin. Am J Infect Control. 1989;17:117.

45. Chang Y-Y, Chuang Y-C, Siu LK, et al. Clinical features of patients with carbapenem nonsusceptible Klebsiella pneumoniae and Escherichia coli in intensive care units: a nationwide multicenter study in Taiwan. J Microbiol Immunol Infect. 2015;48:219-225.

46. Jana M, Misra AK. Synthesis of trisaccharide and a tetrasaccharide repeating unit corresponding to the O-antigen of Shiga toxin producing Escherichia coli 0177. Tetrahedron. 2015;71:3960-3965.

47. Magdy A, Elhadidy M, Abd Ellatif ME, et al. Enteropathogenic Escherichia coli (EPEC): Does it have a role in colorectal tumourigenesis? A Prospective Cohort Study. Int J Surg. 2015;18:169-173.

48. Perepelov A V, Wang Q, Senchenkova SN, et al. Structure and gene cluster of the O-antigen of Escherichia coli O140. Carbohydr Res. 2015;411:33-36.

49. Zdorovenko EL, Golomidova AK, Prokhorov NS, et al. Structure of the O-polysaccharide of Escherichia coli O87. Carbohydr Res. 2015;412:1518.

50. Giakkoupi P, Tryfinopoulou K, Polemis M, et al. Circulation of a multiresistant, conjugative, IncA/C plasmid within the nosocomial Providencia stuartii population in the Athens area. Diagn Microbiol Infect Dis. 2015;82:62-64.

51. Mahrouki S, Chihi H, Bourouis A, et al. Nosocomial dissemination of plasmids carrying blaTEM-24, blaDHA-1, aac(6')-lb-cr, and qnrA6 in Providencia spp. strains isolated from a Tunisian hospital. Diagn Microbiol Infect Dis. 2015;81:50-52.

52. Ovchinnikova OG, Moryl M, Shashkov AS, Chizhov AO, et al. Structure of the O-polysaccharide of Providencia alcalifaciens $\mathrm{O} 2$ containing ascarylose and N-(L-alanyl)-D-glucosamine. Carbohydr Res. 2015;401:11-15.

53. Luzzaro F, Mezzatesta M, Mugnaioli C, et al. Trends in production of extended-spectrum - $\beta$-lactamases among enterobacteria of medical interest: Report of the second Italian nationwide survey. J Clin Microbiol. 2006;44:1659-1664. 